\title{
Hepatitis B Vaccination Status in Dental Practitioners: Local Horrifying Report, National Considerable Issue, and Global Health
}

\section{Impact}

\author{
Mohammad Hosein Amirzade-Iranaq ${ }^{1,2}$, Mehrnaz Hatami ${ }^{3}$, Mohammad Hossein Khosravi $^{2}$ and $^{2}$ \\ Seyed Moayed Alavian (iD) $4,5,{ }^{*}$ \\ ${ }^{1}$ Universal Network of Interdisciplinary Research in Oral and Maxillofacial Surgery (UNIROMS), Universal Scientific Education and Research Network (USERN), Tehran, Iran \\ ${ }^{2}$ Department of Research, Arka Education and Clinical Research Consultants, Tehran, Iran \\ ${ }^{3}$ Department of Prosthodontics, Faculty of Dentistry, Shahid Sadoughi University of Medical Sciences, Yazd, Iran \\ ${ }^{4}$ Baqiyatallah Research Center for Gastroenterology and Liver Diseases (BRCGL), Baqiyatallah University of Medical Sciences, Mollasadra St., Vanak, Tehran, Iran \\ ${ }^{5}$ Middle East Liver Diseases (MELD) Center, Tehran, Iran \\ "Corresponding author: Baqiyatallah Research Center for Gastroenterology and Liver Diseases (BRCGL), Baqiyatallah University of Medical Sciences, Mollasadra St., Vanak, \\ Tehran, Iran. Email: alavian@thc.ir
}

Received 2019 December 18; Revised 2020 December 19; Accepted 2021 January 19.

Keywords: Dentistry, Hepatitis B, Infection Control, Vaccination

\section{Dear Editor,}

Hepatitis B Virus Infection, Epidemiology, And Complication: Hepatitis B Virus (HBV) is a significant global health concern leading to acute and chronic infection. This infection may cause fulminant hepatitis and increase the risk of hepatic decompensation, cirrhosis, and even eventually Hepatocellular Carcinoma (HCC) (1). According to the World Health Organization (WHO), the incidence of individuals infected with HBV is more than two billion worldwide (2). Almost 250 million people are suffering from chronic hepatitis B disease. The mortality of hepatitis B is approximately one million per year (2).

History of HBV Vaccines and Generations: Vaccination is critical for HBV infection control. Three generations of HBV vaccines have been introduced over the years. Krugman's observation about the immunogenicity of HBsAg and the protective effects of the anti-HBS antibody led to the first generation of HBV vaccines, a concentration of plasma driven from $\mathrm{HBV}$-infected individuals containing HBsAg. This vaccine was produced by the Merck and Pasteur institute simultaneously and approved years later by the FDA in 1981. The second generation of HBV vaccines with recombinant DNA technology was prepared using the yeast Saccharomyces cerevisiae, also containing HBsAg. The third-generation vaccines are considerably more immunogenic using pre-S1 and pre-S2 antigens. Recombinant DNA technology was used to manufacture the third-generation vaccines by mammalian cells $(1,3)$.
The prevalence of HBV infection, childhood HCC, and fulminant hepatitis had a remarkable decrease in the USA and some parts of Europe and East Asia after the WHO suggestion (1991) to apply a widespread HBV vaccination in 1997. These days, HBV vaccines are administered three times (repeated on the first and sixth months after first administration) through intramuscular injection. An antiHBs antibody level of more than $10 \mathrm{IU} / \mathrm{L}$ is considered to be an efficient level. A booster dose or revaccination is suggested if the concentration is below $10 \mathrm{IU} / \mathrm{L}$ due to some conditions such as immunosuppression, smoking, obesity, renal failure, and liver disease $(1,4)$.

Cross-infection and Role of Dental Health Care Personnel: In dental care settings, microorganisms can be transmitted in several ways, such as direct contact with patients' oral fluids or blood, indirect contact with contaminated instruments, or environmental surfaces. Also, contact of oral, nasal, or conjunctival mucosa with droplets containing microorganisms is another way of transmission (5). Previous research has shown that HBsAg particles, as well as infectious viruses, can be found in the saliva. However, the number of viruses is low even in HBsAgpositive blood. Anesthetic needles or cutting instruments may cause percutaneous injuries (needle stick), which are the most common occupational exposures in dentistry. The American Dental Association released the results of a survey in which private practitioners reported that they experience an average of 3.2 injuries per year. Also, dental 
educational institutions have reported much higher rates. Considering these reports, the transmission of HBV to the Dental Health Care Personnel (DHCP) is probable. Some previously published studies have reported a prevalence of $16 \%$ to $28 \%$ for HBV serologic markers among dentists. According to available guidelines, only urgent dental care should be rendered for patients with $\operatorname{HBV}(5,6)$. Also, the following steps should be considered:

- Consult the patient's physician regarding the status

- Since hepatitis can alter coagulation, Prothrombin Time (PT) should be measured if bleeding is likely during or after treatment

- Full barrier technique (gloves, masks, disposable gowns, or eye shields) should be used by health care workers who are in direct contact with patients

- Disposable covers are recommended to be used for covering light or drawer handles, as well as bracket trays

- A lined wastebasket should be used for placing all disposable items. Also, guidelines for biohazardous waste should be appropriately followed by bagging and labeling all disposable objects.

- Prevent using ultrasonic instrumentation, highspeed handpieces, or air syringe to reduce aerosol production

- Thirty-second pre-rinsing with chlorhexidine gluconate is recommended.

- All objects and equipment must be scrubbed and sterilized after the procedure is completed.

- A $2 \%$ activated glutaraldehyde solution should be used for wiping all environmental and working surfaces.

Historical cases, including nine clusters of HBV transmission from six infected oral surgeons and three infected general dentists between 1970 and 1987, have attracted more concern towards the DHCP role in cross-infection. Since 1987, no case of such transmissions has been reported, which may be attributable to widespread HBV vaccination in dental healthcare personnel and implementation of the 1991 Occupational Safety and Health Administration (OSHA) Bloodborne Pathogens Standard (5, 7, 8).

Importance of Vaccination in DHCP: In Iran, approximately $2-3 \%$ of people are carriers of the virus, and nearly 300,000 people are suffering from hepatitis, cirrhosis, or hepatocellular carcinoma. In Iran, 70-80\% of chronic hepatitis results from $\mathrm{HBV}$ as the primary cause (2, 9-11).

Carrier DHCPs can normally work with specific cautions and protocols, which is difficult but possible; however, the vaccination paradigm aims to immune as many DHCPs as possible to reduce casualties and scathes. The Center for Disease Control (CDC) reported that 100-200 healthcare personnel died due to workplace-origin hepatitis B. Following an increase in the cases of hepatitis B among healthcare personnel, the workers' protection board declared viral hepatitis an occupational risk. The WHO strategy for viral hepatitis indicates that the goal for incidence is to reduce 30\% and 90\% of new cases with chronic viral hepatitis by 2020 and 2030, respectively. Also, the WHO emphasizes that for enhancement in infection prevention and control in health care settings, it is critical to provide health workers with free immunization against vaccine-preventable diseases, including HBV vaccines, and provide HBV post-exposure prophylaxis as necessary $(2,12-$ 14).

Report of Status of Vaccination in a Medical University: We conducted a pilot study on hepatitis B vaccination in dentistry students of Shahid Sadoughi University of Medical Sciences, Yazd, Iran, in 2017. All dental students who entered the clinical course were asked about hepatitis B vaccination and the check of antibody titration. Of 164 dentistry students, only three (1.9\%) were vaccinated three times, according to the national vaccination guidelines, and 29 (18\%) were not vaccinated at all. Considering the cumulative amount of $82 \%$ of dentistry students were vaccinated one or more times, it was considered that $56.5 \%$ of these students did not check their antibody titration.

Conclusion and Recommendation: Iran's national vaccination guideline comprehensively recommends that all healthcare personnel have to be vaccinated against HBV. Also, healthcare personnel has to check their antibody titration routinely to avoid the transmission of HBV. Our pilot study findings revealed a critical situation that due to the inherent invasive properties of dental procedures, such as extractions and root canal therapies including patient blood encounter, the lack of sufficient vaccination in dental students may cause catastrophic outcomes, affecting patients, DHCP, and their families.

Healthcare policymakers need to consider that although policymaking is necessary, it is more important to make these policies work. A comprehensive monitoring system for controlling healthcare practitioners' vaccination status looks to be the next step to reach a high level of infection immunity in society.

\section{Footnotes}

Authors' Contribution: All authors contributed equally to developing the idea, preparing the draft, and finalizing the manuscript.

Conflict of Interests: None declared by authors.

Ethical Approval: Not applicable.

Funding/Support: None declared by authors. 


\section{References}

1. Rezaee-Zavareh MS, Einollahi B. Hepatitis B vaccination: Needs a revision. Hepat Mon. 2014;14(3). doi:10.5812/hepatmon.17461.

2. WHO. Global hepatitis report 2017. World Health Organization; 2017. Available from: https://www.who.int/hepatitis/publications/globalhepatitis-report2017/en/.

3. World Health O. Hepatitis B vaccines: WHO position paper, July 2017 - Recommendations. Vaccine. 2019;37(2):223-5. doi: 10.1016/j.vaccine.2017.07.046. [PubMed: 28743487].

4. Amirzade-Iranaq MH, Khosravi MH. Infection control status in dental practice: Why to take it serious? Int J Med Rev. 2017;4(1):1-2. doi: 10.29252/ijmr-040101.

5. Younai FS. Health care-associated transmission of hepatitis B \& C viruses in dental care (dentistry). Clin Liver Dis. 2010;14(1):93-104. ix. doi: 10.1016/j.cld.2009.11.010. [PubMed: 20123443].

6. Dahiya P, Kamal R, Sharma V, Kaur S. "Hepatitis" - Prevention and management in dental practice. J Educ Health Promot. 2015;4:33. doi: 10.4103/2277-9531.157188. [PubMed: 26097847]. [PubMed Central: PMC4456879].

7. O'Brien CR, Lim JK. Prevention and management of hepatitis B in healthcare professionals. Curr Hepatol Rep. 2019;18(1):1-8. doi: 10.1007/s11901-019-00444-6.

8. Westin J, Aleman S, Castedal M, Duberg AS, Eilard A, Fischler $\mathrm{B}$, et al. Management of hepatitis B virus infection, updated Swedish guidelines. Infect Dis (Lond). 2020;52(1):1-22. doi: 10.1080/23744235.2019.1675903. [PubMed: 31613181].
9. Babanejad M, Izadi N, Alavian SM. A systematic review and metaanalysis on the prevalence of HBsAg in health care workers from eastern mediterranean and middle eastern countries. Int J Prev Med. 2019;10:144. doi: 10.4103/ijpvm.IJPVM_111_18. [PubMed: 31516685]. [PubMed Central: PMC6716224]

10. Hedayati-Moghaddam MR, Soltanian H, Behzadifar M. Occult hepatitis B virus infection prevalence among different populations of iran: A systematic review and meta-analysis. Hepat Mon. 2020;20(6). doi: 10.5812/hepatmon.101722.

11. Rezaei N, Asadi-Lari M, Sheidaei A, Gohari K, Parsaeian M, Khademioureh S, et al. Epidemiology of hepatitis B in Iran from 2000 to 2016: A systematic review and meta-regression analysis. Arch Iran Med.2020;23(3):189-96. [PubMed: 32126788].

12. WHO. Global health sector strategy on viral hepatitis 2016-2021. Towards ending viral hepatitis. World Health Organization; 2016. Available from: https://www.who.int/hepatitis/strategy2016-2021/ghss-hep/ en/.

13. Schillie S, Vellozzi C, Reingold A, Harris A, Haber P, Ward JW, et al. Prevention of hepatitis B virus infection in the United States: Recommendations of the advisory committee on immunization practices. MMWR Recomm Rep. 2018;67(1):1-31. doi: 10.15585/mmwr.rr6701a1. [PubMed: 29939980]. [PubMed Central: PMC5837403].

14. Coffin CS, Fung SK, Alvarez F, Cooper CL, Doucette KE, Fournier C, et al. Management of hepatitis B virus infection: 2018 guidelines from the Canadian association for the study of the liver and association of medical microbiology and infectious disease Canada. Can Liver J. 2018;1(4):156-217. doi: 10.3138/canlivj.2018-0008. 